\title{
Insegurança alimentar, condições socioeconômicas e indicadores antropométricos em crianças da Região Metropolitana do Rio de Janeiro/Brasil*
}

\author{
Patricia Gomes Pimentel** \\ Rosely Sichieri*** \\ Rosana Salles-Costa****
}

\begin{abstract}
A utilização da Escala Brasileira de Insegurança Alimentar (Ebia) em estudos populacionais no Brasil tem sido estimulada para avaliar a situação de insegurança alimentar (IA), bem como sua associação com indicadores sociais e nutricionais na nossa população. Este estudo foi realizado com o objetivo de avaliar a associação de IA e indicadores antropométricos de crianças menores de 30 meses em Campos Elíseos/Duque de Caxias, Rio de Janeiro. Trata-se de estudo transversal de base populacional, com amostra de 1.085 domicílios, sendo 402 famílias com crianças. A coleta de dados foi realizada de maio a dezembro de 2005. Foram estudados indicadores socioeconômicos e utilizados indicadores antropométricos - peso-idade (P/I), estatura-idade (E/I), pesoestatura $(P / E)$ - para avaliar o estado nutricional. $A$ associação entre as variáveis independente e dependente foi estimada aplicando-se o teste qui-quadrado e o modelo de regressão logística multinomial univariado. A associação entre as medidas antropométricas e IA foi avaliada por meio de modelo de regressão linear univariada. Encontrou-se IA em $72 \%$ dos domicílios. Cerca de $80 \%$ das famílias apresentaram renda familiar mensal per capita inferior a dois terços do salário mínimo. Na análise por regressão logística multinomial, a ausência de saneamento básico e o baixo nível socioeconômico associaram-se a IA moderada e grave, enquanto a presença de aglomerado familiar relacionou-se com IA grave. Renda familiar mensal per capita e escolaridade da pessoa de referência da família associaram-se inversamente com todos os níveis de IA. A insegurança alimentar apresentou associação linear e negativa com escore-z para $P / I(p=0,012)$ e $P / E$ $(p=0,05)$. Este estudo conclui que IA estimada pela Ebia associou-se tanto aos fatores socioeconômicos como à desnutrição infantil. Os resultados sugerem a importância da adoção de políticas públicas com o objetivo de minimizar as diferenças sociais, bem como a situação de IA e fome na população estudada.
\end{abstract}

Palavras-chave: Insegurança alimentar. Indicadores socioeconômicos. Desnutrição infantil.

\footnotetext{
* Este estudo faz parte do projeto Avaliação do estado nutricional, hábitos alimentares e insegurança alimentar no município de Duque de Caxias, Rio de Janeiro: desenvolvimento de um instrumento simplificado para avaliação de consumo alimentar saudável, financiado pelo Conselho Nacional de Desenvolvimento Científico e Tecnológico - CNPq (Processo n0 503139/2003-3) e Instituto Nacional de Câncer (Inca/MS).

** Nutricionista, mestre em Nutrição. Programa de Pós-Graduação em Nutrição - Instituto de Nutrição Josué de Castro UFRJ; Unidade Integrada de Saúde Hamilton Land (Secretaria Municipal de Saúde do Rio de Janeiro).

${ }_{* * *}$ Epidemiologista, professora adjunta do Instituto de Medicina Social da UERJ, pós-doutora em Epidemiologia. Universidade do Estado do Rio de Janeiro, Instituto de Medicina Social, Departamento de Epidemiologia. Núcleo de Epidemiologia e Biologia da Nutrição (Nebin).

${ }_{* * * \star}^{*}$ Nutricionista, professora adjunta do Instituto de Nutrição Josué de Castro da UFRJ, pós-doutora em Saúde Coletiva. Instituto de Nutrição Josué de Castro/Departamento de Nutrição Social e Aplicada/UFRJ.
} 


\section{Introdução}

No mundo 850 milhões de pessoas passam fome, das quais 300 milhões são crianças. Diante deste panorama, a Organização das Nações Unidas (ONU) realizou, em setembro de 2000, a Assembleia do Milênio, onde foi apresentado um conjunto de metas para o desenvolvimento e a erradicação da pobreza: "Objetivos do desenvolvimento do milênio" (ONU, 2000).

No Brasil, de acordo com a Pesquisa Nacional por Amostra de Domicílios (PNAD), realizada em 2004, 72 milhões de pessoas convivem com a experiência de passar fome associada à falta de recursos para aquisição de alimentos.

Neste contexto, cabe enfatizar o tema Segurança Alimentar e Nutricional (SAN), cujo conceito amplo foi discutido na II Conferência Nacional de Segurança Alimentar e Nutricional (CNSAN) realizada em março de 2004, sendo definido como:

\begin{abstract}
Segurança alimentar e nutricional (SAN) a realização do direito de todos ao acesso regular e permanente a alimentos de qualidade, em quantidade suficiente, sem comprometer o acesso a outras necessidades essenciais, tendo como base práticas alimentares promotoras de saúde, que respeitem a diversidade cultural e que sejam social, econômica e ambientalmente sustentáveis (CONSEA, 2007).
\end{abstract}

Esse conceito contempla de forma universal a questão alimentar e foi instrumental para o desenvolvimento da Escala Brasileira de Insegurança Alimentar (Ebia) (PEREZ-ESCAMILLA et al., 2004). A Ebia baseou-se na escala utilizada pelo Departamento de Agricultura dos Estados Unidos da América (BICKEL et al., 2000), criada a partir do estudo qualitativo desenvolvido por pesquisadores da Universidade de Cornell em Nova York (RADIMER et al., 1992), que avaliaram a insegurança alimentar por meio da percepção da fome entre mulheres pertencentes à parcela da população menos favorecida economicamente e de outras escalas, como a do Community Childhood Hunger Identification Project -CHIP (WEHLER et al., 1992). Como resultado, pesquisadores norte-americanos desenvolveram e validaram uma escala com 18 questões, a qual passou a ser utilizada mensalmente em pesquisa telefônica nacional e também nos levantamentos periódicos de Saúde e Nutrição nos Estados Unidos (BARTFELD; DUNIFON, 2005).

A escala brasileira foi desenvolvida por pesquisadores da Universidade de Campinas (Unicamp) e do Observatório de Políticas de Segurança Alimentar e Nutrição da Universidade de Brasília (UNB), além de outras instituições (Universidades Federais da Paraíba e do Mato Grosso e Instituto de Pesquisas da Amazônia), com o apoio técnico e financeiro dos Ministérios da Saúde e do Desenvolvimento Social e Combate à Fome e da Organização Panamericana de Saúde (Opas).

No Brasil, essa escala foi adaptada e validada para a realidade brasileira, entre 2003 e 2004, e utilizada para classificar a população residente em áreas rurais $\mathrm{e}$ urbanas, a partir de pesquisas de natureza qualitativa e quantitativa, em cidades de quatro macrorregiões do Brasil - Norte, Nordeste, Centro-Oeste e Sudeste - e em áreas rurais das cinco macrorregiões brasileiras (PEREZ-ESCAMILLA et al., 2004). O estudo resultou em questionário, de fácil aplicação, considerado adequado para avaliação de insegurança alimentar em inquéritos populacionais nacionais e em pesquisas locais.

Este é o único instrumento já validado no país, que investiga de forma direta a insegurança alimentar nas famílias por meio das suas percepções da fome (SEGALLCORRÊA, 2007), sendo, assim, importante avaliá-lo na sua associação ou predição do estado nutricional infantil. No Brasil, a Ebia foi utilizada no estudo da PNAD (2004), no qual foi possível retratar a distribuição de insegurança alimentar em áreas urbanas e rurais do país. Dados recém-divulgados pela Pesquisa Nacional de Demografia e Saúde (PNDS, 2006), realizada em uma amostra representativa da população feminina do Brasil, em idade reprodutiva, e que utilizou a Ebia como instrumento de análise, revelaram uma proporção de $37,5 \%$ de famílias em situação de insegurança alimentar.

Entretanto, ainda são escassas as publicações relacionando a Ebia e indicadores do estado nutricional de crianças, o que ressal- 
ta a importância da utilização desta escala em estudos de base populacional. Dessa forma, o presente trabalho tem como objetivo avaliar a associação entre indicadores socioeconômicos e insegurança alimentar, bem como a associação entre insegurança alimentar e índices antropométricos de crianças menores de 30 meses.

\section{Material e métodos}

Trata-se de um estudo transversal de base populacional, que faz parte do projeto "Avaliação do estado nutricional, hábitos alimentares e insegurança alimentar no município de Duque de Caxias, Rio de Janeiro", realizado em parceria com o Instituto de Medicina Social da UERJ, Instituto de Nutrição Josué de Castro da UFRJ, Faculdade de Nutrição da UFF, Instituto Nacional de Câncer (MS) e IBGE/Ence.

O Município de Duque de Caxias localiza-se na Região Metropolitana do Rio de Janeiro, a $27 \mathrm{~km}$ de distância da capital do Estado, com uma área de $468,3 \mathrm{~km}^{2}$. Segundo o Centro de Informações de $\mathrm{Da}$ dos do Rio de Janeiro (CIDE, 2005), cerca de $15 \%$ da sua população vive abaixo da linha de pobreza extrema, proporção que corresponde a quase o dobro da média do Estado do Rio de Janeiro (8,69\%); linha da pobreza é um conceito adotado pelo Banco Mundial e que equivale a 1,08 dólar de renda per capita/dia. O segundo distrito de Duque de Caxias (Campos Elíseos) foi selecionado para o presente estudo por ser um dos mais pobres do município. Em 2003 foi considerado o sétimo município com maior índice de pobreza extrema (14,5\%) no Estado do Rio de Janeiro (ROCHA; ALBUQUERQUE, 2003), classificado pelo Índice de Desenvolvimento Humano Municipal (IDH-M) na posição 52 (IDH-M=0,75) no ranking estadual. Sua população residente é de 219.030 habitantes, sendo 15.523 na faixa etária de zero a dois anos (IBGE, 2000).

Utilizou-se amostra probabilística, por conglomerados com três estágios de seleção de domicílios particulares permanentes do segundo distrito do município de Duque de Caxias. No primeiro estágio, foram selecionados 75 setores censitários com probabilidades proporcionais ao tamanho da Base Operacional Geográfica (BOG) de 2000 do IBGE (2000), do total de 322 setores do referido distrito. Buscando aumentar a representatividade da amostra e a precisão das estimativas, além de ampliar a possibilidade de fornecer estimativas para outros domínios de interesse, foi realizada, antes da seleção dos setores, a classificação destes por renda média mensal do responsável pelo domicílio.

Os domicílios dos setores censitários selecionados foram rastreados previamente, para identificar aqueles com crianças na faixa etária de estudo (seis a 30 meses), o que resultou na seleção de 1.125 domicílios, sendo 15 por cada um dos 75 setores censitários, contabilizando uma amostra de 435 domicílios com crianças. Participaram do estudo 1.085 domicílios, com um total de $4 \%$ de perdas (40) (domicílios cujo adulto recusou a visita dos entrevistadores na 1 a ou até a 3 a visita). Dos domicílios com crianças na faixa etária de seis a 30 meses de idade (37\%), 402 participaram do estudo, representando uma perda de $7,6 \%$ em relação ao total de domicílios com crianças amostrados. A coleta de dados foi realizada entre maio e dezembro de 2005. Mais detalhes quanto ao desenho da amostra encontrase publicado em Salles-Costa et al. (2008).

Para avaliação do estado nutricional das crianças, foram tomadas as medidas de peso em gramas, com auxílio de balança pediátrica digital, e comprimento/estatura em centímetros, utilizando infantômetro de madeira portátil, seguindo os procedimentos recomendados pela literatura (LOHMAN, 1992). As medidas comprimento/estatura foram aferidas em duplicata. As crianças foram avaliadas segundo os índices antropométricos peso/idade $(\mathrm{P} / \mathrm{I})$, estatura (comprimento)/idade (E/I) e peso/estatura (comprimento) (P/E), de acordo com o sexo e a faixa etária, utilizando como referência a distribuição proposta pela Organização Mundial de Saúde em 2006 (WHO, 2006). Foram classificadas como eutróficas as crianças cujos índices $\mathrm{P} / \mathrm{I}, \mathrm{E} / \mathrm{I}$ e $\mathrm{P} / \mathrm{E}$ foram maiores ou iguais a $-2,0$ escore-z e, em déficit ponderal e/ou estatural, aquelas que apresentaram valores inferiores a -2,0 
escores-z da mediana da curva de referência. $O$ índice P/E foi utilizado para classificação de sobrepeso, tendo como ponto de corte valores iguais ou superiores a +2 escores-z em relação à estatura (WHO,1995).

Para avaliação das variáveis socioeconômicas, foi aplicado um questionário referente às informações do domicílio (abastecimento de água, coleta de lixo, esgoto sanitário, presença de filtro de água) e da família - renda familiar mensal per capita (total de rendimentos da família dividido pelo número de moradores que dependiam desta renda), escolaridade da pessoa de referência da família, número de moradores por domicílio, raça/cor de pele do chefe/ cônjuge da família (observada pelo entrevistador) e classificação socioeconômica da Abipeme (2006).

As informações sobre insegurança alimentar foram respondidas pelos responsáveis pela alimentação da família, em sua maioria mães das crianças. Para avaliação, utilizou-se a Ebia que se propõe a captar as percepções e algumas dimensões da insegurança alimentar. Esse instrumento - composto por 15 perguntas centrais fechadas com respostas sim ou não referentes aos últimos três meses - reflete a preocupação de a comida acabar antes de se poder comprar mais até a ausência total, podendo afetar todos ou algum membro da família. Das 15 questões, sete dizem respeito a membros da família menores de 18 anos. Cada resposta afirmativa do questionário de insegurança alimentar corresponde a "um ponto", sendo o somatório final da pontuação classificado nas categorias: insegurança leve (1-5 pontos); insegurança moderada (6-10 pontos); e insegurança grave (11-15 pontos) (MARÍN-LEÓN et al., 2005).

$\mathrm{Na}$ análise estatística da associação entre indicadores socioeconômicos e insegurança alimentar, utilizaram-se o teste Qui-quadrado e a regressão logística multinomial univariada, para estimar os valores de razões de chance (Odds ratio) e respectivos intervalos de confiança (IC 95\%), considerando as categorias de desfecho da classificação de insegurança alimentar de acordo com a Ebia, tendo com categoria de referência segurança alimentar.
Para avaliar a associação de insegurança alimentar e o estado nutricional, dado que as prevalências de desnutrição não são de grande magnitude, utilizou-se modelo de regressão linear, tendo como variável independente a pontuação final de cada domicílio pela Ebia expressa como variável contínua, independente dos pontos de corte para classificação dos níveis de insegurança alimentar e, como variáveis dependentes, os valores de escores-z para P/I, E/I e P/E.

Todas as estimativas incorporaram os fatores de expansão, considerando-se as perdas em relação ao total de domicílios estimados, ponderados pela faixa de renda de cada setor e faixa etária estudada, tendo como base as estimativas do IBGE (2000). O banco de dados foi duplamente digitado no programa CSPro 2.5 (CSPRO, 2005), sob supervisão da equipe de pesquisadores, e todos os procedimentos de análise foram realizados no programa Stata 9.0. Este estudo foi aprovado pelo Comitê de Ética e Pesquisa da Universidade do Estado do Rio de Janeiro em 2004.

\section{Resultados}

A amostra estudada foi constituída por 402 crianças, sendo $204(51,7 \%)$ do sexo masculino e 198 (49,3\%) do feminino. Os dados da Tabela 1 mostram que $72,0 \%$ das famílias com crianças de seis a 30 meses de idade avaliadas apresentaram insegurança alimentar, segundo a classificação da Ebia, sendo $12,0 \%$ consideradas com insegurança alimentar grave. Quanto às condições de saneamento básico, verifica-se que 32,3\% dos domicílios não estavam ligados à rede pública de abastecimento de água, 34,1\% não tinham acesso a tratamento público de esgoto e $42,8 \%$ não possuíam filtro para tratamento da água para consumo individual. Entre as famílias estudadas, $77,0 \%$ dispunham de renda familiar mensal per capita inferior a 2/3 do salário mínimo vigente na época da coleta dos dados ( $R \$ 300,00)$, $51,5 \%$ dos chefes das famílias tinham menos de oito anos de estudo, pela classificação Abipeme (2006), 41,8\% das famílias foram classificadas como pertencentes à classe D e $40,8 \%$ dos domicílios amostrados abri- 
TABELA 1

Prevalência dos níveis de insegurança alimentar (1) e caracterização dos domicílios com crianças de seis a 30 meses de idade

Distrito de Campos Elíseos, do Município de Duque de Caxias (RJ) - 2005

\begin{tabular}{|c|c|c|}
\hline Variáveis & $\%(2)$ & IC $95 \%$ \\
\hline \multicolumn{3}{|l|}{ Níveis de insegurança alimentar } \\
\hline Segurança alimentar & $\begin{array}{l}20,0 \\
39,0\end{array}$ & $\begin{array}{l}21,9-34,1 \\
32,4-45,0\end{array}$ \\
\hline Insegurança alimentar leve & 21,0 & $16,4-26,3$ \\
\hline Insegurança alimentar moderada & 12,0 & $7,4-15,6$ \\
\hline \multicolumn{3}{|l|}{ Insegurança alimentar grave } \\
\hline \multicolumn{3}{|l|}{ Saneamento Básico } \\
\hline Lixo com coleta pelo serviço público & 67,7 & $57,9-77,5$ \\
\hline Água fornecida pelo serviço público & 57,2 & $50,9-63,5$ \\
\hline Presença de filtro para tratamento de água & 65,9 & $56,2-75,6$ \\
\hline Esgoto tratado pelo serviço público & 00,9 & \\
\hline \multicolumn{3}{|l|}{ Renda familiar mensal per capita em quartis } \\
\hline De 0 a 50,00 reais & 24,5 & $18,2-30,7$ \\
\hline De 50,10 a 105,00 reais & 23,5 & $17,7-29,4$ \\
\hline De 105,10 a 200,00 reais & 29,0 & $22,2-35,3$ \\
\hline Acima de 200,00 reais & 23,0 & $16,3-29,8$ \\
\hline \multicolumn{3}{|c|}{ Escolaridade da pessoa de referência da família } \\
\hline Analfabeto & 3,1 & $1,4-4,9$ \\
\hline Ensino fundamental incompleto ( $<8$ anos) & 51,5 & $42,9-60,0$ \\
\hline Ensino fundamental completo (8-10 anos) & 23,9 & $18,8-28,9$ \\
\hline Ensino médio completo (11-14 anos) & 20,9 & $14,5-27,3$ \\
\hline Superior ( $\geq 15$ anos) & 0,4 & $0,1-10,0$ \\
\hline \multicolumn{3}{|l|}{ Classificação Abipeme (3) } \\
\hline A & 1,3 & $-0,2-2,8$ \\
\hline B & 4,2 & $1,9-6,5$ \\
\hline $\mathrm{C}$ & 39,3 & $31,9-46,6$ \\
\hline D & 41,8 & $33,9-49,7$ \\
\hline $\mathrm{E}$ & 13,2 & $8,2-18,2$ \\
\hline \multicolumn{3}{|l|}{ Número de pessoas por domicílio } \\
\hline De 1 a 4 & 51,3 & $45,9-56,8$ \\
\hline De 5 a 8 & 40,8 & $35,0-46,6$ \\
\hline Mais de 8 & 7,7 & $4,0-11,4$ \\
\hline \multicolumn{3}{|c|}{ Raça/cor de pele da pessoa de referência ou cônjuge da família (4) } \\
\hline Branca & 28,4 & $22,5-34,3$ \\
\hline Preta/parda & 71,6 & $65,6-77,4$ \\
\hline
\end{tabular}

Fonte: Pesquisa Insegurança Alimentar em Duque de Caxias, Rio de Janeiro, 2005. IMS/UERJ, INJC/UFRJ. Elaboração própria. (1) Segundo a Escala Brasileira de Insegurança Alimentar (Ebia). (2) Valores corrigidos pelo desenho da amostra. (3) Classificação socioeconômica da população apresentada por meio de cinco classes, de acordo com uma pontuação determinada. (4) Heteroclassificação avaliada pelos entrevistadores, considerando a classificação do IBGE.

gavam cinco ou mais moradores. Quanto à raça/cor de pele referente ao chefe/cônjuge da família, $28,0 \%$ das famílias foram consideradas brancas.

Em relação ao perfil nutricional, a prevalência de déficit estatural foi a maior $(8,6 \%)$, seguida por sobrepeso (6,5\%). Não foi observada diferença significativa entre sexos (Tabela 2).

Maiores prevalências de insegurança alimentar foram encontradas nos domicílios onde não havia coleta de lixo regular, naqueles sem ligação à rede pública para tratamento do esgoto, sem filtro para tratamento de água para consumo e sem acesso à rede pública de abastecimento de água. Renda familiar mensal per capita, escolaridade da pessoa de referência da família e classificação Abipeme (2006) foram inversamente e significativamente associadas segundo os níveis de insegurança alimentar (Tabela 3). Entre os domicílios que apresentaram de cinco a oito moradores na composição familiar, a maior prevalência foi para IA leve $(42,7 \%)$, porém, cabe destacar que nos domicílios com mais de oito moradores observou-se prevalência de $28,8 \%$ para IA grave, 4,4 vezes maior do que nos domicílios com um a quatro moradores (6,5\%). Não foi observada associação estatisticamente significativa entre e raça/cor da pele e abastecimento de água com os níveis de insegurança alimentar.

No modelo de regressão logística multinomial univariada (Tabela 4), observou-se que a ausência de saneamento básico associou-se a uma chance maior do que 4 vezes mais de insegurança alimentar 
grave para ausência tanto de coleta de lixo quanto de filtro. Renda familiar mensal per capita e escolaridade da pessoa de referência da família permaneceram asso- ciadas inversamente com todos os níveis de insegurança alimentar $(p<0,0001)$. A classificação Abipeme (2006) também apresentou importante associação com os

TABELA 2

Medidas antropométricas, índices antropométricos e prevalências (1) de déficit nutricional e sobrepeso de crianças de seis a $\mathbf{3 0}$ meses de idade, por sexo

Distrito de Campos Elíseos, do Município de Duque de Caxias (RJ) - 2005

\begin{tabular}{lrrrrrr}
\hline Variáveis & \multicolumn{2}{c}{ Meninos } & \multicolumn{2}{c}{ Meninas } & \multicolumn{2}{c}{ Total } \\
\cline { 2 - 7 } & Média & \multicolumn{1}{c}{ IC 95\% (2) } & Média & IC9 5\% (2) & Média & IC 95\% (2) \\
\hline Medidas antropométricas & 11,3 & $10,9-11,6$ & 11,1 & $10,7-11,3$ & 11,2 & $10,9-11,4$ \\
Peso (gramas) & 82,0 & $80,9-83,0$ & 81,4 & $80,3-82,5$ & 81,7 & $80,9-82,5$ \\
Comprimento (cm) & 19,3 & $18,2-20,3$ & 20,1 & $19,0-21,2$ & 19,7 & $18,9-20,5$ \\
Idade (meses) & & & & & & \\
Índices antropométricos (escores-z) & & & & & \\
Peso/idade & 0,03 & $-0,15-0,22$ & 0,22 & $0,01-0,43$ & 0,12 & $-0,01-0,29$ \\
Estatura/idade & $-0,34$ & $-0,54-0,14$ & $-0,25$ & $-0,49-0,01$ & $-0,29$ & $-0,45-0,14$ \\
Peso/estatura & 0,29 & $0,06-0,53$ & 0,47 & $0,28-0,66$ & 0,38 & $0,23-0,53$ \\
Prevalências (\%) & & & & & & \\
Déficit ponderal (peso/idade) (3) & 1,9 & $0,1-3,6$ & 3,7 & $0,01-7,4$ & 2,8 & $0,7-4,8$ \\
Déficit estatural (estatura/idade) (4) & 10,1 & $5,1-15,0$ & 7,0 & $2,6-11,5$ & 8,6 & $5,3-11,8$ \\
Emaciação (peso/estatura) (5) & 4,5 & $0,5-9,7$ & 2,2 & $0,0-4,5$ & 3,3 & $0,5-6,2$ \\
Sobrepeso (peso/estatura) (5) & 6,3 & $2,8-9,7$ & 6,7 & $2,4-10,9$ & 6,5 & $3,6-9,4$ \\
\hline
\end{tabular}

Fonte: Pesquisa Insegurança Alimentar em Duque de Caxias, Rio de Janeiro, 2005. IMS/UERJ, INJC/UFRJ. Elaboração própria.

(1) Valores corrigidos pelo desenho da amostra. (2) Intervalo de confiança de 95\%. (3) Índice peso-para-idade, considerando déficit valores < -2 escores z. (4) Índice estatura-para-idade, considerando déficit valores < -2 escores z. (5) Índice peso-para-estatura, considerando déficit valores $<-2$ escores $z$ e com sobrepeso valores $\geq+2$.

TABELA 3

Prevalência dos níveis de insegurança alimentar (1), segundo indicadores socioeconômicos das famílias com crianças de seis a $\mathbf{3 0}$ meses de idade

Distrito de Campos Elíseos, do Município de Duque de Caxias (RJ) - 2005

Em porcentagem

\begin{tabular}{|c|c|c|c|c|c|}
\hline Indicadores socioeconômicos & $\begin{array}{l}\text { Segurança } \\
\text { alimentar } \\
\text { (2) }\end{array}$ & $\begin{array}{l}\text { Insegurança } \\
\text { alimentar } \\
\text { leve (2) }\end{array}$ & $\begin{array}{l}\text { Insegurança } \\
\text { alimentar } \\
\text { moderada (2) }\end{array}$ & $\begin{array}{l}\text { Insegurança } \\
\text { alimentar } \\
\text { grave (2) }\end{array}$ & $\begin{array}{l}\text { p-valor } \\
\left(X^{2}\right)(3)\end{array}$ \\
\hline Saneamento básico & & & & & 0,006 \\
\hline \multicolumn{6}{|l|}{ Coleta de lixo regular } \\
\hline Sem coleta & 15,5 & 28,7 & 32,1 & 23,7 & \\
\hline Com coleta & 30,3 & 40,5 & 19,6 & 9,6 & \\
\hline Esgotamento sanitário & & & & & 0,03 \\
\hline Sem rede pública & 27,7 & 28,0 & 27,5 & 16,8 & \\
\hline Com rede pública & 28,5 & 44,3 & 18,3 & 8,9 & \\
\hline Filtro de água & & & & & 0,003 \\
\hline Sem filtro & 18,8 & 37,6 & 26,3 & 17,3 & \\
\hline Com filtro & 35,3 & 39,7 & 17,7 & 7,3 & \\
\hline Abastecimento de água & & & & & 0,62 \\
\hline Sem rede pública & 24,5 & 39,7 & 21,5 & 14,3 & \\
\hline Com rede pública & 30,2 & 38,1 & 21,4 & 10,3 & \\
\hline $\begin{array}{l}\text { Renda familiar per capita mensal } \\
\text { (em reais) (4) }\end{array}$ & & & & & $<0,0001$ \\
\hline De 0 a 50,00 & 5,8 & 42,9 & 27,6 & 23,7 & \\
\hline De 50,10 a 105,00 & 14,8 & 29,1 & 39,8 & 16,3 & \\
\hline De 105,10 a 200,00 & 32,1 & 49,1 & 13,7 & 5,1 & \\
\hline Acima de 200,00 & 61,0 & 31,5 & 5,6 & 1,9 & \\
\hline
\end{tabular}


(...continuação)

\begin{tabular}{|c|c|c|c|c|c|}
\hline Indicadores socioeconômicos & $\begin{array}{c}\text { Segurança } \\
\text { alimentar } \\
\text { (2) }\end{array}$ & $\begin{array}{c}\text { Insegurança } \\
\text { alimentar } \\
\text { leve (2) }\end{array}$ & $\begin{array}{c}\text { Insegurança } \\
\text { alimentar } \\
\text { moderada (2) }\end{array}$ & $\begin{array}{c}\text { Insegurança } \\
\text { alimentar } \\
\text { grave (2) }\end{array}$ & $\begin{array}{l}\text { p-valor } \\
\left(X^{2}\right)(3)\end{array}$ \\
\hline $\begin{array}{l}\text { Escolaridade da pessoa de referência da } \\
\text { família }\end{array}$ & & & & & $<0,0001$ \\
\hline Analfabeto & 13,1 & 33,9 & 21,8 & 31,2 & \\
\hline Ensino fundamental incompleto ( $<$ 8anos) & 15,1 & 41,2 & 25,4 & 18,3 & \\
\hline Ensino fundamental completo (8-10 anos) & 35,8 & 34,3 & 24,7 & 5,2 & \\
\hline Ensino médio completo (11-14 anos) & 51,0 & 41,2 & 6,6 & 1,2 & \\
\hline Superior ( $\geq 15$ anos) & 100 & 0 & 0 & 0 & \\
\hline Classificação (Abipeme) (5) & & & & & 0,001 \\
\hline A & 69,0 & 31,0 & 0 & 0 & \\
\hline B & 54,4 & 39,7 & 5,9 & 0 & \\
\hline C & 37,6 & 47,2 & 9,6 & 5,5 & \\
\hline D & 21,4 & 40,0 & 25,4 & 13,2 & \\
\hline E & 9,3 & 21,2 & 44,1 & 25,4 & \\
\hline $\mathrm{N}^{0}$ de moradores por domicílio & & & & & 0,05 \\
\hline De 1 a 4 & 34,5 & 38,4 & 20,6 & 6,5 & \\
\hline De 5 a 8 & 21,2 & 42,7 & 21,5 & 14,6 & \\
\hline Mais de 8 & 24,0 & 20,8 & 26,4 & 28,8 & \\
\hline $\begin{array}{l}\text { Raça/cor de pele da pessoa de referência/ } \\
\text { cônjuge da família (6) }\end{array}$ & & & & & 0,30 \\
\hline Branca & 31,8 & 42,9 & 17,5 & 7,8 & \\
\hline Preta/Parda & 25,7 & 37,8 & 23,1 & 13,4 & \\
\hline
\end{tabular}

Fonte: Pesquisa Insegurança Alimentar em Duque de Caxias, Rio de Janeiro, 2005. IMS/UERJ, INJC/UFRJ. Elaboração própria. (1) De acordo com a Escala Brasileira de Insegurança Alimentar. (2) Valores corrigidos pelo desenho da amostra. (3) Teste Quiquadrado, sendo o nível de significância menor que $5 \%$ (p-valor <0,05). (4) Os valores estão apresentados em quartis de renda. (5) Classificação socioeconômica da população apresentada por meio de cinco classes de acordo com uma pontuação determinada.

(6) Heteroclassificação avaliada pelos entrevistadores de acordo com a classificação do IBGE.

TABELA 4

Odds ratios (OR) e intervalos de confiança (IC 95\%) entre variáveis socioeconômicas e ambientais e insegurança alimentar de acordo com a Ebia (1). Modelo de regressão logística multinomial com segurança alimentar como variável dependente (categoria de referência=segurança alimentar) Distrito de Campos Elíseos, do Município de Duque de Caxias (RJ) - 2005

\begin{tabular}{|c|c|c|c|}
\hline \multirow[b]{2}{*}{ Variáveis } & \multicolumn{3}{|c|}{ Insegurança alimentar (2) } \\
\hline & $\begin{array}{c}\text { Leve } \\
\text { OR (IC 95\%) }\end{array}$ & $\begin{array}{l}\text { Moderada } \\
\text { OR (IC 95\%) }\end{array}$ & $\begin{array}{c}\text { Grave } \\
\text { OR (IC 95\%) }\end{array}$ \\
\hline \multicolumn{4}{|l|}{ Saneamento básico } \\
\hline \multicolumn{4}{|l|}{ Coleta de lixo } \\
\hline Com coleta & 1,00 & 1,00 & 1,00 \\
\hline Sem coleta & $1,37(0,50-3,79)$ & $3,18(1,37-7,38)$ & $4,85(1,72-13,66)$ \\
\hline \multicolumn{4}{|l|}{ Esgoto sanitário } \\
\hline Com rede pública & 1,00 & 1,00 & 1,00 \\
\hline Sem rede pública & $0,65(0,28-1,51)$ & $1,55(0,69-3,49)$ & $1,94(0,78-4,48)$ \\
\hline \multicolumn{4}{|l|}{ Filtro de água } \\
\hline Com filtro & 1,00 & 1,00 & 1,00 \\
\hline Sem filtro & $1,77(0,83-3,77)$ & $2,77(1,25-6,14)$ & $4,47(1,66-12,02)$ \\
\hline $\begin{array}{l}\text { Renda familiar mensal per capita (em reais) da } \\
\text { pessoa de referência da família ( } 2 \text { ) }\end{array}$ & $0,42(0,29-0,60)$ & $0,26(0,16-0,41)$ & $0,18(0,11-0,30)$ \\
\hline \multicolumn{4}{|l|}{ Escolaridade da pessoa de referência da família } \\
\hline Menos de 7 anos & 1,00 & 1,00 & 1,00 \\
\hline Mais de 7 anos & $0,52(0,34-0,77)$ & $0,34(0,22-0,53)$ & $0,15(0,08-0,29)$ \\
\hline Classificação Abipeme (3) & $1,50(0,90-2,48)$ & $4,46(2,30-8,66)$ & $4,72(2,27-9,84)$ \\
\hline \multicolumn{4}{|l|}{$\mathrm{N}^{\circ}$ de moradores por domicílio } \\
\hline $\begin{array}{l}\text { De } 1 \text { a } 4 \\
5 \text { ou mais }\end{array}$ & $\begin{array}{c}1,00 \\
1,30(0,69-2,44)\end{array}$ & $\begin{array}{c}1,00 \\
155(0.83-2,89)\end{array}$ & $\begin{array}{c}1,00 \\
3,05(1,49-6,24)\end{array}$ \\
\hline
\end{tabular}

Fonte: Pesquisa Insegurança Alimentar em Duque de Caxias, Rio de Janeiro, 2005. IMS/UERJ, INJC/UFRJ. Elaboração própria. (1) Escala Brasileira de Segurança Alimentar. Valores corrigidos pelo desenho da amostra. (2) Variável contínua. (3) Classificação socioeconômica da população apresentada por meio de cinco classes de acordo com uma pontuação determinada. 
TABELA 5

Associação entre insegurança alimentar classificada pela Ebia (1) e escores-z em crianças de seis a 30 meses de idade Distrito de Campos Elíseos, do Município de Duque de Caxias (RJ) - 2005

\begin{tabular}{|c|c|c|c|c|c|c|c|c|c|}
\hline \multirow{3}{*}{ Ebia } & \multicolumn{9}{|c|}{ Escores-z } \\
\hline & \multicolumn{3}{|c|}{ Peso/idade } & \multicolumn{3}{|c|}{ Estatura/idade } & \multicolumn{3}{|c|}{ Peso/estatura } \\
\hline & B & IC 95\% (2) & p-valor & B & IC 95\% (2) & $\mathrm{p}$-valor & B & IC $95 \%(2)$ & p-valor \\
\hline $\begin{array}{l}\text { Insegurança } \\
\text { alimentar }\end{array}$ & $-0,04$ & $-0,07--0,009$ & 0,012 & $-0,03$ & $-0,07-0,0006$ & 0,09 & $-0,03$ & $-0,06-0,0006$ & 0,05 \\
\hline
\end{tabular}

Fonte: Pesquisa Insegurança Alimentar em Duque de Caxias, Rio de Janeiro, 2005. IMS/UERJ, INJC/UFRJ. Elaboração própria. (1) Escala Brasileira de Insegurança Alimentar. Valores corrigidos pelo desenho da amostra. (2) Intervalo de confiança de $95 \%$.

níveis moderado e grave de insegurança alimentar, com valor de razões de chance próximo de cinco. Famílias compostas por cinco moradores ou mais se associaram à insegurança alimentar grave.

$\mathrm{Na}$ análise da associação entre insegurança alimentar em escala contínua com os índices antropométricos (Tabela 5), observou-se associação negativa e significativa entre a pontuação da Ebia com os valores de escores-z para $P / l(p=0,005)$ e $P / E(p=0,05)$.

\section{Discussão}

Em estudo realizado nos Estados Unidos em 2006, utilizando um instrumento similar à Ebia, observou-se insegurança alimentar para $11,2 \%$ das famílias, entre as quais cerca de $4 \%$ apresentaram relato de fome, ou seja, insegurança alimentar grave de acordo com Bartfeld et al. (2005). Nesse mesmo estudo, famílias com crianças registraram prevalência de $16,7 \%$ de insegurança alimentar, sendo 3,8\% com insegurança alimentar grave, situação que tem se mantido ao longo do tempo (NORD et al., 2006). No Brasil, a PNAD, realizada pelo IBGE, que avaliou segurança alimentar e nutricional a partir da Ebia, encontrou uma prevalência de 10,3\% para o nível de insegurança alimentar grave em domicílios com moradores menores de quatro anos de idade. Essa proporção foi de 5,3\% na Região Sudeste e 3,7\% no Estado do Rio de Janeiro (IBGE, 2004).

Em estudos de base populacional realizados por Leão et al. (2005) em Brasília e por Panigassi et al. (2005) em Campinas, os resultados de insegurança alimentar grave foram de 7,7\% e 6,6\%, respectivamente. Em Campos Elíseos, área escolhida para este estudo, observou-se prevalência maior de famílias em situação de insegurança alimentar. Ainda com relação aos dados da PNAD realizada em 2004 (IBGE, 2004), a proporção de domicílios no Brasil em condição de IA moderada ou grave foi 2,5 vezes maior nos domicílios com mais de sete moradores em comparação àqueles com até três moradores. No presente estudo esta proporção foi de 4,4 vezes, evidenciando a gravidade da situação de Campos Elíseos, em especial nas famílias com muitos componentes.

A proporção elevada de domicílios com insegurança alimentar em Campos Elíseos pode ser associada, também, às desigualdades sociais em relação ao acesso aos serviços de saneamento básico no nosso país, como também aos bens de consumo. De acordo com o Relatório de Desenvolvimento Humano (RDH) de 2006, enquanto cerca de $90 \%$ da população tem acesso à água potável no Brasil, proporção semelhante à de países com alto IDH, como Coreia do Sul (92\%) e Cuba (91\%), a taxa de atendimento de coleta de esgoto é de $75 \%$, percentual inferior aos do Paraguai (80\%) e México (79\%). Neste relatório, foi possível observar que, apesar do aumento da proporção de brasileiros com acesso à água potável entre 1990 e 2004, de 83\% para $90 \%$, com relação ao esgoto sanitário não houve evolução neste período.

Para as Metas do Milênio, a Organização das Nações Unidas (ONU) estabeleceu um percentual de $92 \%$ para a população brasileira com água potável em suas residências e $86 \%$ dos domicílios com tratamento sanitário até 2015 (PANIGASSI, 2005; RELATÓRIO 
DO DESENVOLVIMENTO HUMANO, 2006). Os resultados encontrados no presente estudo confirmam a importância de investimento na estrutura de saneamento básico.

Renda familiar mensal per capita e escolaridade da pessoa de referência da família apresentaram forte associação com todos os níveis de insegurança alimentar, como observado nos estudos de Nnakwe et al. (2002) e Bartfeld et al. (2005). Assim como a baixa renda, o menor acesso aos bens de consumo pela classificação Abipeme (2006) revelou-se um importante indicador de insegurança alimentar, principalmente das formas mais graves.

Outro importante resultado observado neste estudo foi a associação entre insegurança alimentar e índices antropométricos, pois crianças pertencentes às famílias com algum nível de insegurança alimentar apresentaram maior déficit ponderal e de estatura, reforçando a possível capacidade da Ebia de predição de desnutrição. Resultado semelhante foi observado em estudo realizado entre famílias com crianças de seis a dezoito meses afro-colombianas residentes em Guapi (Colômbia), em que 14,3\% das crianças das famílias com insegurança alimentar grave apresentavam déficit estatural (ALVARADO et al., 2005). Cabe acrescentar que em estudo realizado na mesma amostra por Barroso et al. (2008), não foi observada associação significativa entre os índices antropométricos e as variáveis socioeconômicas utilizadas, de modo que, no presente trabalho, os modelos lineares não foram ajustados para estas variáveis.

Uma possível limitação deste estudo refere-se à possibilidade de associação entre as variáveis socioeconômicas estudadas, por se tratar de uma população de baixa renda, de modo que o uso de modelos ajustados não permite identificar o efeito isolado de cada variável em relação ao desenlace em questão. Para minimizar este efeito, optou-se pelo modelo logístico multinomial univariado, com o propósito de avaliar as associações brutas entre as variáveis socioeconômicas selecionadas e a situação de insegurança alimentar na população de Campos Elíseos. Trata-se da primeira abordagem sobre a situação de insegurança alimentar desta população e os resultados encontrados são importantes, sugerindo o uso de outros modelos de análise para estimar o quanto cada variável socioeconômica se associa com a insegurança alimentar isoladamente e/ou combinadas entre si.

Infelizmente, os resultados apresentados revelam que a população de estudo, mesmo pertencendo a um município que abriga mais de 10.000 estabelecimentos comerciais e 809 indústrias, além da segunda maior refinaria de petróleo do país, possuindo o sexto maior Produto Interno Bruto (PIB) (1999-2003) no ranking nacional e a segunda maior arrecadação de ICMS do Estado do Rio de Janeiro (IBGE, 1999-2003), apresenta indicadores socioeconômicos desfavoráveis, revelando a má distribuição de renda, as desigualdades e a exclusão social mesmo em áreas urbanas metropolitanas, que têm sido menos assistidas pelos programas governamentais.

Os resultados deste trabalho reforçam a importância de melhores investimentos do capital proveniente tanto das indústrias como do comércio local, na área estudada. A capacitação por meio de aumento na oferta de cursos profissionalizantes para os adultos da região, direcionados ao polo industrial de Campos Elíseos, poderá contribuir para o aumento nos rendimentos familiares.

Quanto às políticas públicas do governo federal, o aumento no número de famílias de Campos Elíseos cadastradas em Programas de Transferência Condicionada de Renda, que no nosso país é representado pelo Programa Bolsa Família (PBF), tende a ser uma alternativa emergencial para minimizar os agravos nutricionais associados à insegurança alimentar, principalmente entre famílias com crianças na faixa etária estudada. Recentemente, estudo realizado entre famílias brasileiras beneficiadas pelo PBF revelou que o acesso ao programa possibilitou o aumento no consumo de vários grupos de alimentos, principalmente entre as famílias em situação de insegurança alimentar (SEGALL-CORREA; SALLESCOSTA, 2008). Cabe ressaltar que em 2005, durante a realização deste trabalho, apenas $11 \%$ de famílias que residiam em Campos 
Elíseos eram beneficiadas pelo PBF, apesar dos indicadores de pobreza na região.

Por fim, as informações sobre os níveis de insegurança alimentar avaliada pela Ebia,

\section{Referências}

ALVARADO, B.E.; ZUNZUNEGUI, M. V.; DELISLE, H. Validación de escalas de seguridad alimentaria y de apoyo social en una población afro colombiana: aplicación en el estúdio de prevalencia del estado nutricional en niños de 6 a 18 meses. Cad. Saúde Pública, 21 (3): 724-36, 2005.

ASSOCIAÇÃO BRASILEIRA DE INSTITUTOS DE PESQUISA DE MERCADO (Abipeme). Classificação socioeconômica critério Abipeme. Disponível em: <http: www.ufrn. $\mathrm{br} /$ sites/fonaprace/perfil_anexo3.doc $>$. Acesso em: 25 maio 2006.

BARROSO, G. S.; SICHIERI, R.; SALLESCOSTA, R. Fatores associados ao déficit nutricional em crianças residentes em uma área de prevalência elevada de insegurança alimentar. Rev. Brasileira de Epidemiologia, 11(3):484-494, 2008.

BARTFELD, J.; DUNIFON, R. State-level predictors of food insecurity and hunger among households with children. USDA (United States Department of Agriculture), October, 2005. Disponível em: <http://www. ers.usda.gov>. Acesso em: 19 dez. 2005.

BICKEL, G.; NORD, M.; PRICE, C.; HAMILTON, W.; COOK, J. Measuring food security in the United States: guide to measuring household food security. Alexandria: Office of Analysis, Nutrition, and Evaluation, U.S.Department of Agriculture, 2000.

CENSUS AND SURVEY PROCESSING SYSTEM - CSPRO, version 3.2. International Programs Center, U.S. Census Bureau: Washington, USA, 2005.

CENTRO DE INFORMAÇÕES E DADOS DO RIO DE JANEIRO (CIDE). Diagnóstico do mercado de trabalho formal do Estado do Rio de Janeiro. Disponível em: <http:// www.cide.rj.gov.br/pnb/pages/munic_dcax. htm >. Acesso em: 20 nov. 2005.

CONSELHO NACIONAL DE SEGURANÇA ALIMENTAR. Princípios e diretrizes de uma bem como sua associação com os indicadores socioeconômicos e antropométricos das crianças, reforçam sua utilidade na identificação de áreas vulneráveis no país.

política de segurança alimentar. Brasília: Editora Positiva, 2004.

CONSELHO NACIONAL DE SEGURANÇA ALIMENTAR E NUTRICIONAL (CONSEA). Documento base da III Conferência Nacional de Segurança Alimentar e Nutricional. Brasília: Consea, 2007.

INSTITUTO BRASILEIRO DE GEOGRAFIA E ESTATÍSTICA. Censo Demográfico 2000. Rio de Janeiro, IBGE, 2001.

Pesquisa Nacional por Amostra de Domicílios - Segurança Alimentar, 2004. Disponível em: < http://www.ibge.gov. br/>. Acesso em: 26 set. 2006.

Produto Interno Bruto dos Municípios 1999-2003. Disponível em: <http://www.ibge.gov.br/>. Acesso em: 24 abr. 2005.

LEÃO, M. M. Fome e insegurança alimentar no Distrito Federal. Dissertação (Mestrado em Nutrição Humana). Brasília, Faculdade de Ciências da Saúde, Universidade de Brasília, 2005.

LOHMAN, T. G. Advances in body composition. Human Kinectics, Champaing, Illinois, 1992.

MARÍN-LEÓN, L.; SEGALL-CORRÊA, A. M.; PANIGASSI, G.; MARANHA, L. K.; SAMPAIO, A. F. A.; PÉREZ-ESCAMILLA, R. A percepção de insegurança alimentar em famílias com idosos em Campinas, São Paulo, Brasil. Cad. Saúde Pública, 21:1.433-40, 2005.

NNAKWE, N.; YEGAMMIA, C. Prevalence of food insecurity among households with children in Coimbatore, India. Nutrition Research, 22:1.009-16, 2002.

NORD, M.; ANDREWS, M.; CARLSON, S. Measuring food security in the United States. USDA (United States Department of Agriculture). November, 2006. Diponível em: <http://www.ers.usda.gov>. Acesso em: 21 nov. 2006. 
ORGANIZAÇÃO DAS NAÇÕES UNIDAS. Projeto do Milênio das Nações Unidas. Investindo no desenvolvimento: um plano prático para atingir os objetivos de desenvolvimento do milênio. Visão geral. Disponível em: <http://www.pnud.org. $\mathrm{br} / \mathrm{milenio} /$ arquivos/resumodoprojeto $>$. Acesso em: 20 jan. 2007.

PANIGASSI, G. Inquérito populacional sobre a percepção da segurança alimentar intrafamiliar no município de Campinas, SP. Tese (Doutorado). Campinas, Faculdade de Ciências Médicas, Universidade Estadual de Campinas, 2005.

PEREZ-ESCAMILLA, R.; SEGALL-CORREA, A. M.; MARANHA, L. K.; SAMPAIO, M. F.; MARINLEON, L.; PANIGASSI, G. An adapted version of the U.S. Department of Agriculture Food Insecurity Module is a valid tool for assessing household food insecurity in Campinas, Brazil. J. Nutr. 134(8):1923-8, 2004.

PESQUISA NACIONAL DE DEMOGRAFIA E SAÚDE DA CRIANÇA E DA MULHER/PNDS 2006. Relatório técnico. Brasília/DF, 2008. Disponível em: <http://portal.saude.gov.br/ portal/arquivos/pdf/PNDS.pdf > . Acesso em: 11 jul. 2008.

RADIMER, K. L.; OLSON, C. M.; GREENE, J. C.; CAMPBELL, C. C.; HABICHT, J. P. Understanding hunger and developing indicators to assess it in women and children. J. Nutr. Educ, 24(1 suppl.): 36- 4, 1992.

RELATÓRIO DO DESENVOLVIMENTO HUMANO 2006. Brasil deve cumprir tranquilamente meta de água e com dificuldade a de saneamento. Disponível em: <http:// www.undp.org/hdr2006>. Acesso em: 04 mai. 2007.
ROCHA, S.; ALBUQUERQUE, R. C. Seminário especial fome e pobreza. Geografia da pobreza extrema e vulnerabilidade à fome. Estudos e pesquisas, 2003.

SALLES-COSTA, R.; PEREIRA, R. A.; VASCONCELLOS, M. T. L.; VEIGA, G. V.; MARINS, V. M. R. et al. Associação entre fatores socioeconômicos e insegurança alimentar: estudo de base populacional na Região Metropolitana do Rio de Janeiro, Brasil. Revista de Nutrição, 21(supl.): 99-109s, 2008.

SEGALL-CORRÊA, A. M. Insegurança alimentar medida a partir da percepção das pessoas. Estudos Avançados, 21(60): 143-154, 2007.

SEGALL-CORRÊA, A. M.; SALLES-COSTA, R. Novas possibilidades de alimentação a caminho? Democracia Viva, 39 (jun): 6873, 2008.

STATA CORPORATION: version 9.0. Texas: college Station, 2006.

WEHLER, C. A.; SCOTT, R. I.; ANDERSON, J. J. The community childhood identification project: a model of domestic hungerdemonstration project in Seattle, Washington. J. Nutr. Ed., 24:29-35, 1992.

WORLD HEALTH ORGANIZATION (WHO). Child growth standards. Methods and development: World Health Organization, 2006. Disponível em: <www.who.int/ childgrowth/en/>. Acesso em: 20 out. 2006.

The use and interpretation of anthropometry physical status: the use and interpretation of anthropometry. Report of a WHO expert committee. Geneva, World Health Organization, 1995 (WHO Technical Report Series, n. 854).

\section{Resumen}

Inseguridad alimentaria, condiciones socioeconómicas e indicadores antropométricos en niños de la región metropolitana de Río de Janeiro/Brasil

La utilización de la Escala Brasileña de Inseguridad Alimentaria (Ebia) en estudios poblacionales en Brasil, ha sido estimulada para evaluar la situación de inseguridad alimentaria (IA), así como su asociación con indicadores sociales y nutricionales en nuestra población. Este estudio fue realizado con el objetivo de evaluar la asociación de IA e indicadores antropométricos de niños menores de 30 meses en Campos Elíseos/Duque de Caxias, Río de Janeiro. Se trata de un estudio transversal de base poblacional, con una muestra de 1.085 hogares, siendo 402 
las familias con niños. La recolección de datos fue realizada de mayo a diciembre de 2005. Fueron estudiados indicadores socioeconómicos y utilizados indicadores antropométricos peso-edad $(P / I)$, estatura-edad $(E / I)$, peso-estatura $(P / E)$ - para evaluar el estado nutricional. La asociación entre las variables independiente y dependiente fue estimada aplicándose el test chi-cuadrado y el modelo de regresión logística multinomial univariada. La asociación entre las medidas antropométricas e IA fue evaluada por medio del modelo de regresión lineal univariada. Se encontró IA en el $72 \%$ de los hogares. Cerca del $80 \%$ de las familias presentaron un ingreso familiar mensual per cápita inferior a los dos tercios del salario mínimo. En el análisis por regresión logística multinomial, la ausencia de saneamiento básico y el bajo nivel socioeconómico se asociaron a una IA moderada y grave, mientras que la presencia de aglomerado familiar se relacionó con una IA grave. El ingreso familiar mensual per cápita y la escolaridad de la persona de referencia de la familia se asociaron inversamente con todos los niveles de IA. La inseguridad alimentaria presentó una asociación lineal y negativa con escore-Z para $P / I(p=0,012)$ y $P / E(p=0,05)$. Este estudio concluye que la IA estimada por la Ebia se asoció tanto a los factores socioeconómicos como a la desnutrición infantil. Los resultados sugieren la importancia de la adopción de políticas públicas con el objetivo de minimizar las diferencias sociales, así como la situación de IA y hambre en la población estudiada.

Palabras-clave: Inseguridad alimentaria. Indicadores socioeconómicos. Desnutrición infantil.

\section{Abstract}

Food insecurity, socioeconomic conditions and anthropometric indicators in children living in the Metropolitan Region of Rio de Janeiro, Brazil

The Brazilian Food Insecurity Scale has been valuable in demographic studies in Brazil for evaluating the situation of food insecurity and its association with social and nutritional indicators in the country's population. This present study was carried to evaluate the association of food insecurity with anthropometric indicators of children under 30 months of age in the Campos Eliseos neighborhood of the city of Duque de Caxias, in the greater Rio de Janeiro region. It was a demographically based cross-section study on a sample of 1,085 households, 402 of which consisted of families with children. The data was gathered between May and December of 2005. Socioeconomic indicators were studied and anthropometric indicators (age-weight and age-height) were used to appraise the subjects' nutritional state. The association between the independent and dependent variables was determined by applying the Q-square test and the multinomial-univariate logistics regression model. The association between the anthropometric data and food insecurity was determined by a linear univariate regression model. Food insecurity was found in $72 \%$ of the households, and approximately $80 \%$ of the families showed monthly family per capita income lower than two thirds of the already low minimum wage. In the analysis by multinomial logistics regression, the lack of basic water supply and sanitation and the low socioeconomic level were associated with moderate and serious food insecurity, whereas the presence of agglomerated families was related to serious food insecurity. Monthly per capita family income and the educational level of the reference person in the family were inversely associated with all levels of food insecurity. Food insecurity presented a linear and negative association with score-z for age-weight $(p=0.012)$ and for height-weight $(p=0.05)$. This study concludes that food insecurity as measured by the Ebia is associated with both socioeconomic factors and child malnutrition. The findings suggest the importance of establishing public policies aimed at minimizing social differences and the situation of food insecurity and hunger in the population studied.

Keywords: Food insecurity. Socioeconomic indicators. Child malnutrition.

Recebido para publicação em 18/05/2009. Aceito para publicação em 29/07/2009. 